\title{
Variations
}

Variations

Revue internationale de théorie critique

$17 \mid 2012$

Critique du travail

\section{Roland Pfefferkorn, Genre et rapports sociaux de sexe}

\section{Viviane Albenga}

\section{(2) OpenEdition}

Journals

Édition électronique

URL : http://journals.openedition.org/variations/415

DOI : 10.4000/variations.415

ISSN : 1968-3960

Éditeur

Les amis de Variations

\section{Référence électronique}

Viviane Albenga, «Roland Pfefferkorn, Genre et rapports sociaux de sexe », Variations [En ligne], 17 |

2012, mis en ligne le 15 octobre 2012, consulté le 22 septembre 2020. URL : http://

journals.openedition.org/variations/415; DOI : https://doi.org/10.4000/variations.415

Ce document a été généré automatiquement le 22 septembre 2020.

Les ami•e•s de Variations 


\title{
Roland Pfefferkorn, Genre et rapports sociaux de sexe
}

\author{
Viviane Albenga
}

\section{RÉFÉRENCE}

Roland Pfefferkorn, Genre et rapports sociaux de sexe, Lausanne, Editions Page-deux, collection Empreinte, 2012

1 Cet ouvrage de Roland Pfefferkorn apporte un éclairage généalogique et théorique sur deux grands concepts issus de la théorie féministe : le genre et les rapports sociaux de sexe. D'une très grande clarté et d'une grande richesse dans les références mobilisées, l'ouvrage met ces concepts en discussion, l'auteur défendant davantage l'apport heuristique des rapports sociaux de sexe sans pour autant négliger de présenter toutes les nuances présentes dans les différentes théories du genre et dans les usages de ce concept. Ce point de vue affirmé avec finesse, argumenté et étayé par des références théoriques et empiriques, donne lieu à une lecture stimulante en ce qu'il explicite un certain nombre de débats au sein des études féministes, et révèle toute l'importance scientifique et politique des enjeux à l'œuvre derrière le choix des concepts. L'ouvrage s'inscrivant dans une collection dédiée à la compréhension du système capitaliste, on ne peut que se réjouir de la place accordée dans ce cadre à la théorie féministe, qui n'est pas seulement un complément à l'analyse des rapports de classe, mais en fait partie intégrante comme le démontre l'auteur.

2 Le tout premier mérite de l'ouvrage réside dans sa présentation des concepts issus du mouvement féministe. Le premier chapitre restitue toute leur importance à des concepts oubliés ou "relégués" à la sphère militante, et qui ont forgé le socle théorique de ce qui deviendra plus tard le concept de « rapports sociaux de sexe»: ainsi le concept de "patriarcat ", centré sur l'analyse de l'économie domestique chez Christine Delphy, ou celui de «sexage» de Colette Guillaumin qui définit l'appropriation de la classe des femmes par celle des hommes, appropriation à la fois 
collective et individualisée par l'entremise du mariage. Le second chapitre sur les définitions plurielles du genre reprend les définitions les plus marquantes de ce concept : le genre désigne le sexe social (John Money, Robert Stoler, Ann Oakley), c'està-dire la construction sociale des identités sexuées; il renvoie à un système patriarcal et fondé sur la norme hétérosexuelle (Gayle Rubin) ; il trouble les catégories binaires et sape les identités, selon Judith Butler, Teresa de Lauretis, Eve Sedgwick, qui déconstruisent la répartition binaire entre homme et femme, entre sexualité hétérosexuelle et homosexuelle; ou enfin, il signifie des rapports de pouvoir (Joan Scott), puisque masculin et féminin sont des catégories utilisées pour soutenir symboliquement d'autres hiérarchies, et sont elles-mêmes l'objet de luttes symboliques pour la définition de la masculinité et de la féminité dominantes à une époque donnée, dans un milieu social donné. Les différentes approches sont présentées de telle sorte que même le lecteur - la lectrice - averti(e) peut y trouver une mise en perspective qui clarifie la polysémie du concept. Dans ce chapitre s'affirme déjà le point de vue de l'auteur, selon lequel le concept de genre se prête à des usages qui négligent voire occultent la dimension matérielle de l'oppression, à la différence du concept de rapports sociaux de sexe. Il souligne ainsi que «la théorie queer est peut-être davantage occupée par des questions de différence culturelle et par l'opposition binaire qui sous-tend l'hétérosexualité normative que par la question de l'oppression. [...] Car les identités, les significations et les subjectivités vécues sont largement contraintes par les contextes culturels et sociaux. » (p. 64) Cette critique peut être affinée en soulignant qu'au-delà de la théorie queer, toute pensée de la subversion du genre ne saurait faire l'économie des ressources matérielles, mais également culturelles, qui rendent la subversion possible et qui sont inégalement distribuées socialement.

Le troisième chapitre est consacré à l'exposition des limites du concept de genre. L'une des limites les plus pertinentes adressée au concept de genre serait qu'il occulte le caractère socialement construit du sexe biologique (démontré notamment par Cynthia Kraus). À cette critique, Christine Delphy avait répondu dans son texte Penser le genre qu'il était nécessaire de garder le concept de genre pour rompre avec le naturalisme, ce que ne permet pas le terme de «sexe " trop connoté de sens commun. La critique de l'institutionnalisation du genre s'avère également nécessaire : « les usages du vocable genre à partir des années 1990 se sont parfois éloignés des intentions critiques des théoriciennes féministes, par-delà leur diversité, et ont contribué à accentuer encore la polysémie du terme » (p. 69.) En effet, les usages institutionnels du genre, qu'ils soient politiques ou scientifiques, voire certains usages sociologiques, ont pu contribuer à affaiblir la charge critique du genre: ce concept ne désigne pas seulement des différences sexuées, ou ne consiste pas seulement à intégrer "les femmes" dans l'analyse, il désigne un rapport de pouvoir fondé sur une binarité arbitraire et hiérarchique entre masculin et féminin. Là où la démonstration paraît un peu moins convaincante, c'est lorsque l'auteur souligne que les études de genre se sont parfois centrées sur les représentations et les discours, sans mentionner les travaux en question. Plus encore, peut-on dire que le genre "gomme» les rapports de classe, comme il l'évoque en prenant appui sur des exemples d'enquêtes sociologiques qui ne se situent pas dans la perspective d'une sociologie des classes sociales? Il prend certes la précaution de noter que "l'évacuation du conflit et des rapports sociaux peut aussi prendre appui sur l'émergence du genre. Mais le concept [...] est alors manifestement instrumentalisé » (p. 84). On pourrait peut-être avancer que ce n'est pas le concept de genre qui occulte en lui-même les rapports de classe, mais il ne contraint pas à les 
prendre nécessairement en compte dans toute analyse, à l'inverse du concept de rapports sociaux de sexe qui est fondé sur la co-construction des rapports de domination.

4 À ce titre, le dernier chapitre plaide pour l'intérêt heuristique des rapports sociaux de sexe, un concept étroitement articulé à la « division sexuelle du travail ». Selon Danièle Kergoat, les rapports sociaux désignent des antagonismes entre des groupes autour d'enjeux centraux, le travail - entendu au sens large comme " production du vivre » - et le contrôle de la procréation. En ce sens, les rapports sociaux de classe et de sexe ne peuvent être appréhendés séparément. Il en va de même des rapports sociaux de " race", pris en compte beaucoup plus récemment dans la sociologie française. Ces différents rapports sociaux peuvent être définis séparément pour l'analyse, mais empiriquement ils se construisent réciproquement. Plus exactement, ils sont « consubstantiels » et « coextensifs »: ils se produisent les uns les autres et il ne saurait être déterminé de variable en dernière instance. La centralité du travail dans les processus de domination, mais aussi d'émancipation, est défendue avec force par R. Pfefferkorn : «objectivement, socialement et matériellement, c'est le travail, c'est-àdire l'organisation et la division du travail, qui sert de levier à la domination du groupe des hommes sur celui des femmes. Et c'est pourquoi il est l'enjeu des rapports sociaux de sexe.» (p. 115).

Bien qu'argumenté, ce point de vue paraît discutable à plusieurs titres : en premier lieu, il sous-estime l'enjeu spécifique du contrôle des corps et de la procréation. Puisque l'ouvrage se clôt sur des exemples de luttes de femmes au travail, on ne saurait oublier que l'une des principales luttes féministes a pris, et prend toujours comme objet, le droit à disposer de son corps, notamment par l'accès à l'avortement libre et gratuit. Michèle Ferrand, sociologue qui a contribué à l'atelier Production/Reproduction du CNRS, a mis en évidence avec Nathalie Bajos l'existence d'une "nouvelle norme procréative» qui rend compte de la force des injonctions à la maternité pour les femmes françaises d'aujourd'hui ${ }^{1}$. Les rapports sociaux de sexe sont constitués par ces enjeux qui sont difficiles à englober dans la définition du « travail ». On peut également prendre l'exemple des violences conjugales qui sont statistiquement moins fréquentes dans les couples dont les deux membres travaillent, mais qui existent significativement dans toutes les classes. En ce sens, ce que Colette Guillaumin désignait par l'appropriation de la classe des femmes va au-delà des enjeux strictement matériels. Ainsi, défendre une approche matérialiste de la domination ne signifie pas que les bases symboliques de celle-ci soient secondaires, comme l'auteur le reconnait lui-même dans une phrase qui mériterait des développements empiriques : « Un rapport d'exploitation peut être structuré par un rapport de domination et ce dernier peut porter sur des dimensions matérielles ou idéelles. » (p. 21) Dans cette perspective, on peut rappeler la parution récente en français du Contrat sexuel de Carole Pateman, qui démontre que le patriarcat a des bases politiques, car l'exploitation économique des femmes est rendue possible par leur subordination préalable ${ }^{2}$. Mais au-delà de ces éléments de discussion, demeure l'intérêt vivace d'une remise en perspective dynamique du genre et des rapports sociaux de sexe, qui peut ranimer des débats scientifiques féconds autour de ces concepts, loin d'une utilisation consensuelle et mécanique. 


\section{NOTES}

1. Nathalie Bajos et Michèle Ferrand, «L'interruption volontaire de grossesse et la recomposition de la norme procréative ", Sociétés contemporaines 1/2006 ( $\left.{ }^{\circ} 61\right)$, p. 91-117.

2. Carole Pateman, Le Contrat sexuel, La Découverte/Institut Émilie-du-Châtelet, 2010.

INDEX

Mots-clés : critique du travail, correspondance, artistes, socialisme vert, travailleurs culturels, Wertkritik, Gorz André, dialogue, Vincent Jean-Marie, écologie, modèles alternatifs, Marx Karl, penser le Capital, ethos du travail, Eglise Orthodoxe, salariat, domination

\section{AUTEURS}

\section{VIVIANE ALBENGA}

Post-doctorante à l'ENS de Lyon, laboratoire Triangle, ANR "Genre et violences à l'école" 